\title{
Alternatives of Revenue for Corrective Actions of Wind Generators in a Delegated Dispatch
}

\author{
Edgardo D. Castronuovo* and Julio Usaola* \\ \{ecastron, jusaola\}@ing.uc3m.es \\ * Department of Electrical Engineering, Universidad Carlos III de Madrid \\ Av. de la Universidad, 30 - 28911 Leganés (Madrid) - Spain
}

\begin{abstract}
Face to critical contingence situations, the System Operator can require wind farms output reductions. The zonal Delegated Dispatches can act as interface between the System Operator and the producers, allowing a faster and accurate answer. Three probable approaches are mathematically formulated and analyzed in the paper, separately considering the controllability and the possibility of disconnecting the wind farms. The methods are tested and compared by using a realistic test network, extracted from the Spanish national network.
\end{abstract}

Index Terms - Wind Generation, Generation Dispatch, Delegated Dispatch, Optimization, Nonlinear Programming, Mixed Integer Programming.

\section{INTRODUCTION}

The production of a wind parks depends of installed capacity, available wind power and controllability. For large penetration of the Wind Generation (WG) in the system, the operation of the system could become a complex task. In Spain, WG responds for 11,200 MW of installed capacity, of a total of $82,300 \mathrm{MW}$ in the power system (2006 data) [1]. Today, 13,6\% of the installed capacity corresponds to this conventionally nondispatchable production and this percentage is increasing yearly.

Face to contingence situations in the system operation, the System Operator (SO) has the alternative of disconnect some wind parks. However, to select and to disconnect the appropriate WG in a contingence situation requires several assessments and communications between the SO and each individual wind park. Delegated Dispatches (DD) are supposed to be zonal intermediate entities, connecting the wind farms of a region with the SO. Therefore, the DD aims to obtain the best operation point for the wind parks of a region, regarding the limitations imposed by the SO.

In [2], the action of a DD is analyzed. The optimal operation point of each individual producer is obtained through optimization techniques, considering the best wind parks profit and observing the restrictions forced by the contingence situation. In [3], the proposed method is extended and examined in a realistic case. Particular considerations are performed in relation to the optimal both active and reactive control actions allowed to each individual wind producer. The present paper analyses three different options to share the reduction in the total of active power production in the DD: proportional, considering controllability prices and taking in consideration both controllability and interruptive prices. The alternatives are evaluated in a realistic case, extracted from the Spanish National grid.

\section{The Delegate Dispatches}

When no other possibilities are allowed and to avoid a contingence situation, the wind parks can be switched off at the point of interconnection with the system, regarding the security of the system. Wind power is produced in wind farms of a size up to $50 \mathrm{MW}$, generally concentrated in zones of high wind potential. Therefore, the selection of the WG that may be disconnected in a contingence situation is a hard mission for the SO, requiring numerous communications with the individual wind parks. Some power system operators (like Red Eléctrica de España, REE) aims to delegate the control of the WG in contingence situations to zonal DDs. When a reduction of the WG production is required to lead with a contingence situation, the SO communicates to the DD the maximum input of WG allowed in the DD controlled region. The $\mathrm{DD}$ are the midpoint between the $\mathrm{SO}$ and the individual wind farms, determining the best operation point of each particular wind producer.

The zonal DD has, at least, the following advantages: better communication channels with the wind parks, more accurate previsions for each individual wind farm and a better knowledge of the power controllability options of the parks. Old wind generators have not active power controllability, only allowing the full connection/disconnection of the wind farm. New wind generators (especially doubly fed induction ones) can fully control both active and reactive productions, following a wanted production profile [4-6]. The DD can use this ability of the new wind generators to improve the whole operation, sending operational points considering interruptive and controllability aptitudes of the producers.

Preliminary technical tests in real cases for the DD action in Spain are being executed in the first quarter of 2007 and the full implementation of the first DDs are expected throughout 2007. The regulation about DD in Spain is still under process, and the rules not yet fully developed. In the present paper, the current proposals of the Spanish SO about the actions required from a DD are considered, together with propositions of some wind producers. The mathematical formulations, cost structures, analyses and tests are responsibility of the authors. 


\section{Alternatives for the Optimal Allocation of THE REDUCTIONS IN CONTINGENCE SiTUATIONS}

In the following sections, three alternatives for the optimal allocation of the reductions required by the SO are formulated: proportional, taking in consideration controllability prices and considering both controllability and interruptive prices.

\section{A. Proportional Repartition of the Reductions}

This is the simplest share of the reductions in the revenue, considering the diminution actions a common service required for the system operation. Only generators with ability to control the active power production can participate in this repartition. If there are old technology wind generators in the DD area, these generators will pay to the controllable wind power producers for the controllability service.

The proportional reduction can be formulated through an optimization problem, as showed in (1)-(11).

$$
\begin{array}{ll}
\text { Max } & P R \\
\text { s.t. } & S_{G j} \cdot \cos \varphi_{j}-P_{G j}^{a v} \cdot P R=0 \\
& P_{\text {out }} \leq P_{\text {out }}^{M a x} \\
& S_{G i} \cdot \cos \varphi_{i}+P_{G i}^{N C}-P_{D i}-P(V, \alpha)=0 \\
& S_{G i} \cdot \sin \varphi_{i}+Q_{G i}^{N C}-Q_{D i}-Q(V, \alpha)=0 \\
& \alpha_{s k}=0 \\
& 0.0 \leq P R \leq 1.0 \\
& S_{G j} \cdot \cos \varphi_{j} \geq 0.0 \\
& \cos \varphi_{i} \geq \cos \varphi_{i}^{\min } \\
& V_{i}^{\min } \leq V_{i} \leq V_{i}^{\max } \\
& T_{i k}^{\min } \leq T_{i k} \leq T_{i k}^{\max } \quad i \neq k \\
& \\
&
\end{array}
$$

Where $P R$ is the proportional reduction coefficient; $S_{G j}$ and $\varphi_{j}$ are the values of apparent power generation and production angles of the controllable generator connected to bus $j ; P_{G j}^{a v}$ is the maximum forecasted production of the wind generator $j$, in the considered period; $P_{G i}^{N C}$ and $Q_{G i}^{N C}$ are the active and reactive productions of noncontrollable wind farms in bus $i$, respectively; $P_{D i}$ and $Q_{D i}$ are active and reactive power demands at bus $i$, respectively; $V_{i}$ and $\alpha_{i}$ are the module and angle of the bus voltage at bus $i$, respectively; $\alpha_{s k}$ is the tension angle at the slack bus; $P_{\text {out }}$ and $P_{\text {out }}^{\text {Max }}$ are total output and maximum total output, respectively, of the active power production in the DD area; $V_{i}^{\min }$ and $V_{i}^{\max }$ are minimum and maximum limits of the tension module in bus $i ; T_{i k}$ is the apparent power transmission between buses $i$ and $j$; $T_{i k}^{\min }$ and $T_{i k}^{\max }$ are the minimum and maximum limits of the transmission line between buses $i$ and $k$, respectively; $m$ is the number of plants with active power controllability in the DD area; and $n$ is the number of buses at the internal of the DD system.

Objective function (1) aspires to maximize the proportional repartition $(P R)$ factor of the wind parks, aiming to reach the maximum active power output authorized by the SO. The proportional repartition factor is the same for all the controllable wind farms.

Equations (2) allow the calculation of $P R$. For all the $m$ controllable wind generators, the proportional participation can be defined as

$$
P R=\frac{S_{G 1} \cdot \cos \varphi_{1}}{P_{G 1}^{a v}}=\frac{S_{G 2} \cdot \cos \varphi_{2}}{P_{G 2}^{a v}}=\ldots=\frac{S_{G m} \cdot \cos \varphi_{m}}{P_{G m}^{a v}}
$$

The definition of a unique variable $P R$ for all the participant generators will guarantee a proportional repartition of the required reductions.

Face to a contingence situation, the SO communicates to the DD the maximum of wind active power output that the system can absorb, $P_{\text {out }}^{\operatorname{Max}}$. The optimization problem must restrict the active power DD injection in the bus of interconnection to the system $\left(P_{\text {out }}\right)$ to accept this contingence restriction, as observed in (3). Regarding the proportional repartition, when the sum of the non-controllable active power producers is greater than the maximum acceptable output communicated by the SO, no allowable operation is possible. This test must be performed before the execution of the optimization problem (1)-(11).

Equality restrictions (4) and (5) represent the balances of both active and reactive powers in the buses of the internal to the DD grid. The wind farms are connected to the output bus (the interconnection with the system point) through transmission lines. The operational set-points calculated by the DD must maintain adequate conditions for the operation in this internal to the DD grid. Equations (4) and (5) correspond to the AC power flow of the internal system. In these equations, both active and reactive power generations of non-controllable wind farms are considered as fixed quantities. As in the conventional AC power flow, the angle of the voltage in the slack bus is zero (6).

The participation factor $(P R)$ can vary among 0 and 1.0 (7). When $P R=0$, the controllable wind parks do not introduce active power to the system. If $P R=1$, no reductions are required by the operation in the DD area.

The injection of active power generation only can be a positive value, as showed in (8). From (2) and (7), the upper limit for the active power production of the wind farm $j$ is $S_{G j} \cdot \cos \varphi_{j} \leq P_{G j}^{a v}$.

Technologically, the lower limit for the power factor $\cos \varphi_{i}$ of each $j$ wind farm must be limited by an inferior bound $\cos \varphi_{i}^{\min }$, as expressed in (9).

Equations (10) and (11) show the operational limits of the voltage in the buses and of the apparent flows in the lines of the internal system, respectively.

The optimization problem (1)-(11) aims to proportionally share the reductions in the production of the wind farms, when a contingence situation requires a decrease in the whole DD generation. The control in the 
active power production of the controllable producers is assumed as common service required by the operation. Therefore, the costs associated for reducing the production are not considered. In the following sections, two approaches to take into account the costs for the reduction service are presented.

\section{B. Reduction Share, considering Controllability Prices}

When the reduction service costs of the producers are known, the controllability service can be offered within the DD. In this case, the optimal operation aims the minimum cost for the reductions, considering the controllability prices, the whole desiderated reduction and the operational restrictions of the system. As in the proportional repartition, the non controllable wind producers must pay for the reduction service provided by the controllable wind parks, after the operation. In equation (12)-(22), the optimization problem formulated by considering controllability prices is presented.

$$
\begin{array}{ll}
\min & \sum_{j=1}^{m} c p_{j} \cdot C R_{j} \\
\text { s.t. } & C R_{j}=P_{G j}^{a v}-S_{G j} \cdot \cos \varphi_{j} \\
& P_{\text {out }} \leq P_{\text {out }}^{\text {Max }} \\
& S_{G i} \cdot \cos \varphi_{i}+P_{G i}^{N C}-P_{D i}-P(V, \alpha)=0 \\
& S_{G i} \cdot \sin \varphi_{i}+Q_{G i}^{N C}-Q_{D i}-Q(V, \alpha)=0 \\
& \alpha_{s k}=0 \\
& 0.0 \leq C R_{j} \leq P_{G j}^{a v} \\
& S_{G j} \cdot \cos \varphi_{j} \geq 0.0 \\
& \cos \varphi_{i} \geq \cos \varphi_{i}^{\min } \\
& V_{i}^{\min } \leq V_{i} \leq V_{i}^{\max } \\
& T_{i k}^{\min } \leq T_{i k} \leq T_{i k}^{\max } \quad i \neq k \\
&
\end{array}
$$

Where $c p_{j}$ and $C R_{j}$ are the controllability price and controllability reduction factor of wind producer $j$, respectively.

If all the DD wind producers offer their prices for the controllability service $(c p)$, the operational point aims to prioritize reductions in producers with lower controllability prices, as showed in (12). The reduction of the active power production $\left(C R_{j}\right)$ of producer $j$ is defined as the difference among the maximum available production $\left(P_{G j}^{a v}\right)$ and the real active production ( $\left.S_{G j} \cdot \cos \varphi_{j}\right)$, (13). The controllability reduction factor must be a positive value, lower than $P_{G j}^{a v}$, as expressed in (18).

When active power output reductions are required, the best economical operation is to inject the maximum amount of allowable active generation to the system (14). As in the proportional repartition, when the sum of the non-controllable active power producers is greater than the maximum acceptable output communicated by the $\mathrm{SO}$, no allowable operation is possible.
In the present formulation, the operational constraints (15)-(17) are similar to the equations (4)-(6) and the restrictions (19)-(22) are equivalent to expressions (8)-(11), respectively.

In the optimization problem (12)-(22), the producers without control of the active power production will offer very high controllability prices.

\section{Reduction Share, considering both Controllability and Interruptive Prices}

When large reductions of the whole DD production are required by the $\mathrm{SO}$, the complete controllability ability of the DD controllable producers could not be sufficient. In these cases, the solution of (12)-(22) will involve reductions of the active power production in some non controllable producer, in spite of the high price submitted by the non controllable wind farms. This solution suggests that one or more than one producers must be interrupted to reach the SO requirements.

The present approach explicitly considers the interruption of wind farms in the mathematical formulation. Moreover, it allows that the non controllable producers can offer this interruptive alternative to the $\mathrm{DD}$, competing with the controllability prices submitted by the controllable producers.

The interruptive option of the producers can be represented through discrete variables. The inclusion of discrete variables and the associated prices in (12)-(22) results in a non-linear mixed integer optimization problem, of complicated solution. However, if the active power losses in the internal losses are small and may be neglected, the internal DD grid may be represented by a linearized model, simplifying the solution. In Spain, most of the wind producers are connected to the system interconnection point through simple circuits. Therefore, in a first approximation, the internal DD grid will be represented by means of a linearized model (DC model).

The proposed optimization problem is showed in equations (23)-(27).

$$
\begin{array}{cl}
\min & \sum_{j=1}^{m}\left(c p_{j} \cdot C R_{j}+i p_{j} \cdot I R_{j} \cdot P_{G j}^{a v}\right) \\
\text { s.t. } & \sum_{j=1}^{m} P_{G j}=P_{\text {out }}^{M a x} \\
& P_{G j}+C R_{j}=P_{G j}^{a v} \cdot\left(1-I R_{j}\right) \\
& I R_{j}+\frac{C R_{j}}{P_{G j}^{a v}} \leq 1.0 \\
& {\left[P_{G i}\right]=[B] \cdot\left[\varphi_{i}\right]}
\end{array}
$$

$$
\begin{aligned}
& i=1 \ldots n \\
& j=1 . . . m
\end{aligned}
$$

Where $i p_{j}$ is the interruptive price of wind producer $j$; $I R_{j}$ is a binary variable, representing the interruptive reduction factor of wind producer $j ; P_{G j}$ is the active power production of wind farm $j ;\left[P_{G i}\right]$ and $\left[\varphi_{i}\right]$ are the vectors of active power generations and angles of the 
voltage in all the $i$ buses; and $[B]$ is the admittance matrix of the DC model [7].

Objective function (23) aims to minimize the regulation cost in the active power reduction process, regarding both interruptive and controllability actions in the DD. The interruptive reduction factor $\left(I R_{j}\right)$ is a binary variable with two values, 0 and 1 . Both controllable and non-controllable wind farms must submit the interruptive and controllability prices to the DD. The wind parks that do not want to control the production will submit a very high controllability price, only participating in the interruptive actions.

As previously, the maximum output in the whole production is approximated to $P_{\text {out }}^{M a x}$. In the linearized model, the losses in the transmission lines are not considered. Therefore, the whole production in the DD can be calculated as the sum of the productions in all the wind farms (24).

The amount generated by each wind producer depends of both interruptive and controllability actions performed by the producer. $I R_{j}$ is a binary variable, assuming only $(0 ; 1)$ values. Therefore, if wind park $j$ is required to interrupt its production $\left(I R_{j}=1\right)$, the upper limit in the active power generation in this period is $\operatorname{zero}\left(P_{G j}^{a v} \cdot\left(1-I R_{j}\right)=0.0\right)$. When the wind park does not interrupt the active power production $\left(I R_{j}=0\right)$, the active power generation $\left(P_{G j}\right)$ can be reducing by increasing the controllability reduction factor $\left(C R_{j}\right)$, as showed in (25).

The controllability actions only can be possible if the wind park is not interrupted $\left(I R_{j}=0\right)$. Consequently,

$$
\begin{aligned}
& \frac{C R_{j}}{P_{G j}^{a v}} \leq\left(1.0-I R_{j}\right) \text { or, as expressed in } \\
& I R_{j}+\frac{C R_{j}}{P_{G j}^{a v}} \leq 1.0 .
\end{aligned}
$$

In equation (27), the linearized model is included in the formulation, calculating the voltage angles in the buses of the internal to the DD system.

The reduction share problem (23)-(27) is formulated through a mixed integer linear optimization problem. In the present paper, this problem is solved by using the solver miqp.m, version 1.6, a Matlab function for solving mixed integer quadratic programs, developed by A. Bemporad and D. Mignone [8].

In the following section, results obtained from a realistic test case are presented.

\section{RESUlts}

To test the different approaches, a typical cluster of wind parks from the Spanish National network is considered. In Table 1, the main characteristics of the system internal to the DD are presented.
TABLE I

TRANSMISSION LINE CHARACTERISTICS

\begin{tabular}{|c|c|c|c|c|c|}
\hline From & To & $\boldsymbol{R}$ [p.u.] & $\begin{array}{c}\boldsymbol{X}_{\boldsymbol{L}} \\
\text { [p.u.] }\end{array}$ & $\begin{array}{c}\boldsymbol{B}_{\boldsymbol{C}} \\
{[\mathbf{p . u .} \text { ] }}\end{array}$ & $\begin{array}{c}\boldsymbol{T}_{\boldsymbol{i} \boldsymbol{j}}{ }^{\max } \\
{[\mathbf{p . u .}}\end{array}$ \\
\hline 1 & 2 & .00141 & .074 & 0.001 & 5.0 \\
\hline 2 & 3 & .01340 & .044 & 0.002 & 5.0 \\
\hline 2 & 6 & .00160 & .1433 & 0.002 & 4.0 \\
\hline 3 & 4 & .00360 & .3145 & 0.001 & 4.0 \\
\hline 3 & 5 & .00170 & .2993 & 0.002 & 4.0 \\
\hline
\end{tabular}

In Table 1 , the p.u. values of the resistance $(R)$, series reactance $\left(X_{L}\right)$, parallel susceptance $\left(B_{C}\right)$ and maximum apparent power flow $\left(T_{i j}{ }^{\max }\right)$ in the transmission lines of the internal grid are presented. To calculate the p.u. values of the table, the apparent power and voltage bases are 100 MVA and $66 \mathrm{kV}$, respectively. In Table 2, the generators description is showed.

TABLE II

GENERATION AND DEMANDS CHARACTERISTICS

\begin{tabular}{|c|c|c|c|c|c|c|}
\hline Bus & $\begin{array}{c}\boldsymbol{P}_{G}{ }^{\max } \\
{[\mathbf{p . u .}]}\end{array}$ & $\begin{array}{c}\text { Avail. } \\
\text { Power } \\
{[\%]}\end{array}$ & $\begin{array}{c}\boldsymbol{P}_{G}{ }^{\boldsymbol{a v}} \\
{[\mathbf{\%} . \mathbf{u} .]}\end{array}$ & $\begin{array}{c}\boldsymbol{B} \\
{[\mathbf{p} . \mathbf{u} .]}\end{array}$ & $\begin{array}{c}\boldsymbol{c p} \\
{[\boldsymbol{\epsilon} /} \\
\mathbf{M W}]\end{array}$ & $\begin{array}{c}\boldsymbol{i p} \\
{[\boldsymbol{G} /} \\
\mathbf{M W}]\end{array}$ \\
\hline 1 & -- & -- & 0.0 & 0.0 & 0 & 0 \\
\hline 2 & 1.5 & 63.0 & 0.945 & 0.09 & 35 & 3.5 \\
\hline 3 & -- & -- & 0.0 & 0.0 & 0 & 0 \\
\hline 4 & 1.4 & 58.0 & 0.812 & 0.06 & 24 & 2.4 \\
\hline 5 & 1.5 & 72.0 & 1.08 & 0.096 & 30 & 3.0 \\
\hline 6 & 1.0 & 67.0 & 0.67 & 0.096 & 39 & 3.9 \\
\hline
\end{tabular}

The second column of Table II shows the installed capacity in the buses of the DD system. The available wind power at the present operation is presented in columns 3 and 4, in percentage and p.u. values, respectively. In column 5 , the susceptance $B$ linked to the bus is showed.

The costs for both controllability and interruptive services are presented in columns 6 and 7 of Table II. At the time of written, there are not DDs in operation in Spain. Therefore, the controllability prices were approximated to those submitted by ordinary generators to the spinning reserve. To show the difference between controllability and interruptive actions, the interruptive price was represented as 0.1 times the respective controllability price.

In the solution of the different approaches, the slack bus is the node 1 , the voltage limits are $(0.95 ; 1.05)$, there are not active $\left(P_{D i}\right)$ or reactive $\left(Q_{D i}\right)$ demands in the internal grid and the lower limit of the transmission lines are equal to the negative of the maximum $\operatorname{limit}\left(T_{i k}^{\min }=-T_{i k}^{\max }\right)$. 


\section{A. Proportional Repartition of the Reductions}

A situation without output restrictions is represented in the optimization problem (1)-(11) by a high output active power $\left(P_{\text {out }}^{\text {Max }}\right)$ limit, such that

$$
P_{\text {out }}^{\text {Max }}>\sum_{j=1}^{m} P_{G j}
$$

In the present case, $P_{\text {out }}^{\text {Max }}=10.0$ is used. When no output restrictions are communicated by the SO to the $\mathrm{DD}$, the wind farms inject to the system the maximum of the available production, as showed in the $4^{\text {th }}$ column of Table II. The total generation is $\sum_{j=1}^{m} P_{G j}=3.507$ p.u.

However, the active power DD output is a lower value, $P_{\text {out }}=3.438$ p.u., because the losses. The active power losses in the internal grid are

$$
\sum_{j=1}^{m} P_{G j}-P_{\text {out }}=3.507-3.438=0.069 \text { p.u. }
$$

Without output restrictions in the active power production, the total of active power losses in the DD grid ( 0.067 p.u.) represents $1.9 \%$ of the generation.

In Table III, the generations in all de buses for a reduction of $20 \%$ in the whole production of the DD $\left(P_{\text {out }}^{\text {Max }}=0.8 \cdot 3.438=2.750\right.$ p.u. $)$ are shown.

TABLE III

GENERATIONS FOR A 20\% REDUCTION OF THE DD PRODUCTION

\begin{tabular}{|c|c|c|c|c|c|c|c|}
\hline & & Bus 1 & Bus 2 & Bus 3 & Bus 4 & Bus 5 & Bus 6 \\
\hline \multirow{2}{*}{$\boldsymbol{P}_{G j}$} & $\mathrm{pu}$ & 0.0 & 0.753 & 0.0 & 0.647 & 0.860 & 0.534 \\
\cline { 2 - 8 } & $\%$ & 0.0 & 20.3 & 0.0 & 20.3 & 20.3 & 20.3 \\
\hline $\boldsymbol{Q}_{G j}$ & $\mathrm{pu}$ & 0.0 & -0.02 & 0.0 & -0.09 & 0.031 & -0.12 \\
\hline
\end{tabular}

To calculate the values of Table III, all the generators of Table II are considered controllable, regarding the active power production. As observed in Table III, the reduction in the active power production of the generators is lightly greater $(20.3 \%)$ than the required whole diminution $(20.0 \%)$. This result is necessary to compensate the reduction in the active power losses, due to the lower flows within the DD.

In Fig. 1, the active power generations in the DD wind farms are depicted, for different values of the output restrictions.

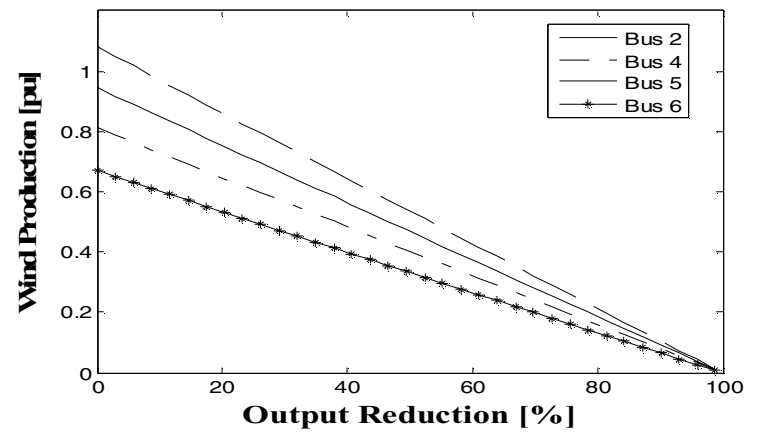

Fig. 1. Wind Production in the Controllable DD Wind Farms, when decreasing the Output Active Power Limit. Proportional Repartition.
When a proportional repartition is used (Fig. 1), the active power reductions are smoothly reduced to 0.0 , for increasing SO reduction requests. From Fig. 1, the optimal active power set-point for the wind producers can be obtained, as a function of the required reduction in the whole DD production. As depicted, when a reduction in the DD production is required by the $\mathrm{SO}$ and $\mathrm{a}$ proportional repartition scheme is adopted, all the controllable generators must vary the operation point, decreasing the active power production.

\section{B. Reduction Share, considering Controllability Prices}

In the reduction share approach considering controllability prices $\left(6^{\text {th }}\right.$ column of Table II), the operation aims to decrease the whole controllability costs of the DD action (12)-(22). As previously, all the generators represented in Table II have active power controllability.

In Fig. 2, the variation of the active power generations for the DD producers for different values of the active power output restriction is showed.

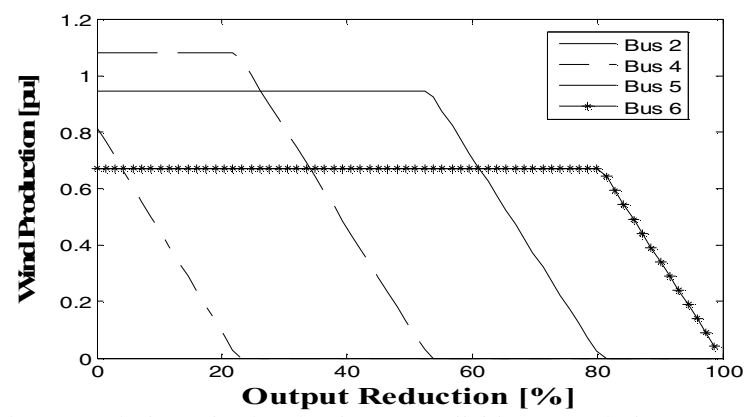

Fig. 2. Wind Production in the Controllable DD Wind Farms, when decreasing the Output Active Power Limit. Only Controllability Prices.

When considering controllability costs in the repartition scheme (Fig. 2), for each percentage of output reduction only one wind producer must decrease partially the active power production, with the others producers either working at full production or disconnected. Between 0.0 and $23 \%$ of whole DD output reduction, the wind farm that offered the low controllability price (the producer at bus 4) will be requested to partially decrease the active power generation to reach the SO appeal. Between 23 and $54 \%$ of active power output reduction, the producer at bus 5 must regulate the own generation to obtain the diminution requested to the DD. In this interval, generators at buses 2 and 6 are working at full production and the wind farm at bus 4 is disconnected. For active power output restrictions between 54 and $81 \%$, the wind park at bus 2 will partially reduce the generation (with producers at buses 4 and 5 disconnected and wind farm at bus 6 untouched). When the SO requests active power reductions greater than $81 \%$ all the wind producers must be disconnected, excepting the wind farm at bus 6 . In these cases, the producer with the larger controllability price will generate all the allowable active power output and the associated losses.

Besides other advantages, the use of controllability prices in the DD operation allows that only one controllable producer is requested to exercise its active 
power control ability in a contingence situation. The others wind producers only must be asked for switch on/off the complete production, a generally simpler procedure than controlling the generation.

\section{Reduction Share, considering both Controllability and Interruptive Prices}

In the present approach, each wind producer must submit two prices for the DD action: the price for interrupting the complete production and the controllability price. Therefore, the non-controllable wind farms can compete with active power controllable producers, aiming the best DD operation. In Fig. 3 to 6 , solutions of optimization problem (23)-(27) for different active power restrictions in the DD output are shown.

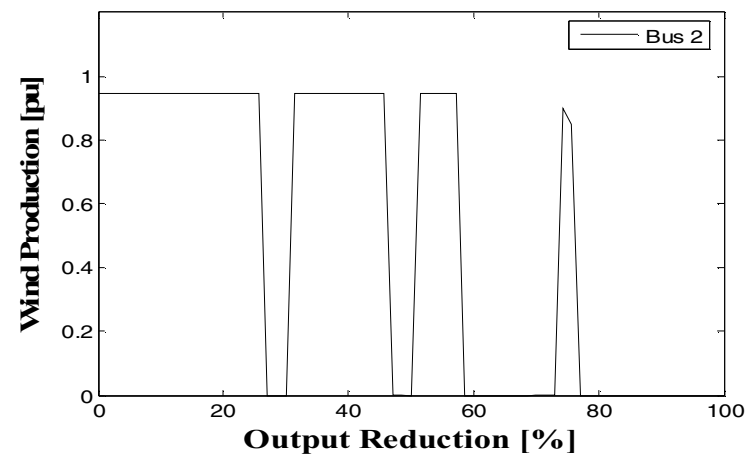

Fig. 3. Wind Production in the Controllable DD Wind Farm at Bus 2 , when decreasing the Output Active Power Limit. Both Controllability and Interruptive Prices.

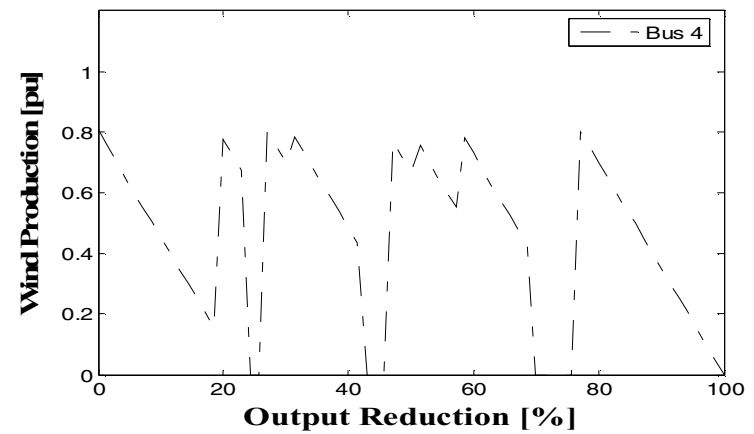

Fig. 4. Wind Production in the Controllable DD Wind Farm at Bus 4, when decreasing the Output Active Power Limit. Both Controllability and Interruptive Prices.

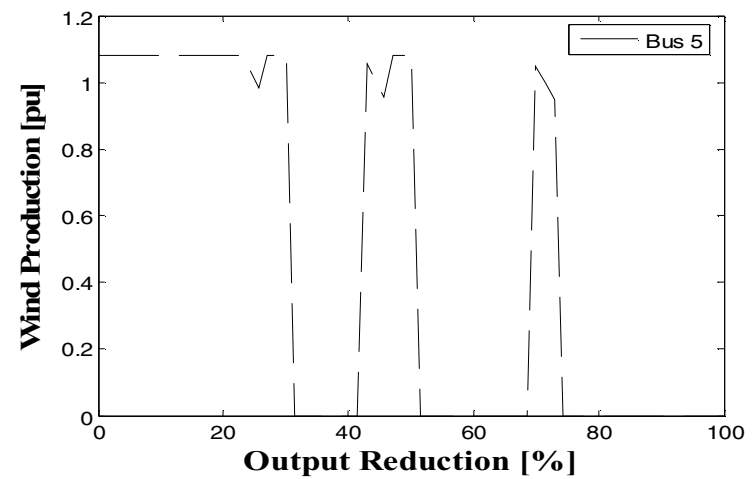

Fig. 5. Wind Production in the Controllable DD Wind Farm at Bus 5, when decreasing the Output Active Power Limit. Both Controllability and Interruptive Prices.

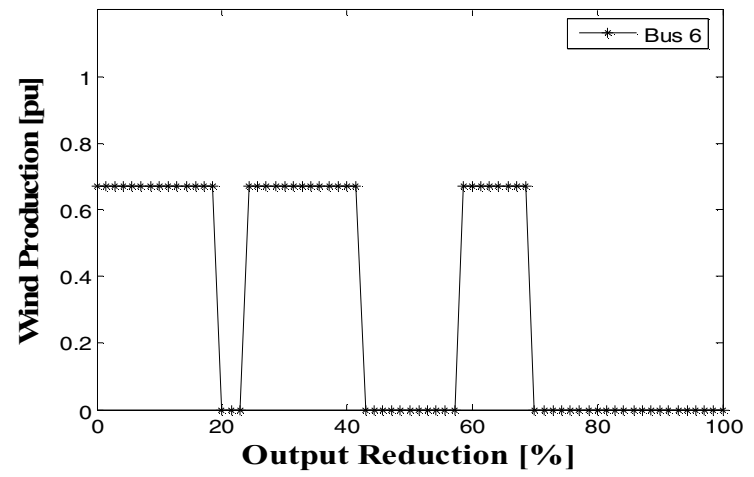

Fig. 6. Wind Production in the Controllable DD Wind Farm at Bus 6 , when decreasing the Output Active Power Limit. Both Controllability and Interruptive Prices.

In Fig. 3 to 6 , the active power generation in the buses with wind farms is depicted. As the interruptive prices $\left(7^{\text {th }}\right.$ column of Table III) are much lower than the controllability costs, for each value of DD active power output reduction the algorithm solution firstly aims the best interruptive combination of wind farms. From this step, switch off signals are sent to some producers. Between the still connected generators, the producer that offered the low controllability price is requested to control the active power production to match the whole DD desiderated generation. As observed in Fig. 6, the producers that offered the largest controllability price (wind farm at bus 6) is not asked to regulate the active power production in the present simulations. However, the interruptive ability of this wind park is utilized to improve the whole DD operation.

\section{CONCLUSIONS}

The better knowledge of the technical abilities of the participating producers allows improving the answer of a wind parks cluster, when facing a contingence. The DD can combine this knowledge with better zone previsions of the prospective wind active productions and enhanced communication channels. The present paper analyses three probable approaches for the DD action, independently considering both controllability and interruptive capacities of the wind farms. Mathematical formulations are proposed and tested in realistic cases.

The proportional repartition of the active power diminution between the DD participants considers the repartition as a common service, necessary for the operation. Therefore, no prices are considered in this approach. For any value of reduction, all the controllable producers are requested to control the production, simultaneously. The non-controllable wind farms can not participate in the contingence response, paying after the operation to the controllable producers for this service.

As an alternative approach, the producers can previously submit to the DD the controllability prices for managing the production. When the SO requires a reduction in the whole active power production, the DD calculate the optimal operational point for each wind farm, sending set-points to the producers. For any value of the whole reduction, only one wind farm is asked for 
controlling its production. The others wind parks must switch on/off the generation to comply the SO requirement, a simpler action than manage the active power production. However, the wind producer that must to control its production can vary in function of the required reduction and the controllability prices. As the participant wind farms can be called to control its production, non-controllable wind farms can not participate in the contingence response.

The consideration of both interruptive and controllability prices results in a more complex optimization problem. Therefore, the present paper uses a simplified formulation for the DD action. As in the previous approach, only one wind park must regulate its production for a specified whole reduction. In the performed simulations, all the wind farms are called to use its interruptive ability. In contrast, only these producers that offered to control its active power production with lower prices are compelled to manage its production. This behaviour allows the participation of non-controllable producers in the DD contingence response.

\section{REFERENCES}

[1] Red Eléctrica de España, El Sistema Eléctrico Español, avance del Informe 2006, available on-line in www.ree.es.

[2] E.D. Castronuovo y J. Usaola, Delegated Dispatch of Wind Generators in Spain, Proc. of the 2006 EWEC, (2006), Athens, Greece.

[3] E.D. Castronuovo and J. Usaola, Optimal Controllability of Wind Generators in a Delegated Dispatch, Electr. Power Syst. Res (in press), (2006) doi:10.1016/j.epsr.2006.10.015

[4] R.G. de Almeida, E. D. Castronuovo and J.A. Peças Lopes, Optimum Generation Control in Wind Parks when Carrying Out System Operator Requests, IEEE Trans. on Power Systems, 21(2), (2006)

[5] J. Ekanayake, L. Holdsworth and N. Jenkins, Control of DFIG Wind Turbines, Power Engineering Journal, vol. 17, No 1, pp. 28-32, February 2003.

[6] J. Ekanayake and N. Jenkins, Comparison of the Response of Doubly Fed and Fixed-Speed Induction Generator Wind Turbines to Changes in Network Frequency, IEEE Transactions on Energy conversion, vol. 19, No. 4, pp. 800-802, December 2004.

[7] A.J. Wood and B.F. Wollenberg, Power Generation Operation and Control, $2^{\text {nd }}$. edition, John Wiley \& Sons, USA, 1984.

[8] A. Bemporad and D. Mignone, miqp.m, A Matlab Function for solving Mixed Integer Quadratic Programs, version 1.6, available on-line in http://control.ethz.ch. 